\title{
Associação entre neuropatia diabética e sintomas de trato urinário inferior nas pessoas com diabetes mellitus: revisão integrativa
}

\author{
Association between diabetic neuropathy and lower urinary tract symptoms in people with \\ diabetes mellitus: integrative review
}
Asociación entre la neuropatía diabética y los síntomas del tracto urinario inferior en personas con diabetes mellitus: revisión integradora

Heloneida Maria Leoncio Mahle Costa ${ }^{1}$, Débora Popovicz ${ }^{1}$, Robson Giovani Paes ${ }^{2 \star}$, Gisela Maria Assis $^{1}$, Tatiane Coradassi Esmanhotto ${ }^{1}$, Ana Rotília Erzinger ${ }^{1}$.

\section{RESUMO}

Objetivo: Investigar a associação entre neuropatia diabética e sintomas do trato urinário inferior nas pessoas com diabetes mellitus. Métodos: Revisão integrativa da literatura, que seguiu seis etapas; a busca ocorreu no mês de outubro de 2019, no Portal da Biblioteca Virtual de Saúde e nas bases de dados National Library of Medicine, Medical Literature Analysis and Retrieval System Online e Scopus, utilizando os descritores e operadores booleanos "urinary incontinence" AND "diabetes mellitus" AND "diabetic neuropathies"; os critérios de inclusão foram publicações de 2009 a 2019, nos idiomas português, inglês e espanhol. Resultados: Dos 52 estudos provenientes da busca, oito compuseram a amostra final; as mulheres foram majoritárias nos estudos; a neuropatia diabética estava associada aos sintomas de trato urinário inferior, sendo a incontinência urinária, incontinência de urgência, noctúria, bexiga hiperativa e disfunção sexual feminina; a obesidade, cor branca, tempo e descontrole da doença foram fatores associados aos sintomas urinários. Considerações finais: A avaliação da sensibilidade protetiva dos pés e detecção precoce de neuropatias, oferece uma oportunidade para a prevenção dos sintomas de trato urinário inferior. As limitações deste estudo foram a heterogeneidade metodológica dos artigos, a estratégia de busca e a falta de dados com relação ao sexo masculino.

Palavras-chave: Incontinência urinária, Diabetes mellitus, Neuropatias diabéticas, Adulto, Sintomas do trato urinário inferior.

\begin{abstract}
Objective: To investigate the association between diabetic neuropathy and lower urinary tract symptoms in people with diabetes mellitus. Methods: Integrative literature review, which followed six steps. The search took place in October 2019, on the Virtual Health Library Portal and the National Library of Medicine, Medical Literature Analysis and Retrieval System Online Scopus databases, using the Boolean descriptors and operators "urinary incontinence", "diabetes mellitus" and "diabetic neuropathies". The inclusion criteria were publications from 2009 to 2019, in Portuguese, English and Spanish. Results: Of the 52 studies from the search, eight made up the final sample and women were the majority in the studies. The diabetic neuropathy was associated with lower urinary tract symptoms, with urinary incontinence, urge incontinence, nocturia, overactive bladder and female sexual dysfunction. Obesity, white race, time and uncontrolled disease were factors associated with urinary symptoms. Final considerations: The assessment of the protective sensitivity of the feet and early detection of neuropathies offers an opportunity for the prevention of lower urinary tract symptoms. The limitations of this study were the methodological heterogeneity of the articles, the search strategy and the lack of data regarding the male gender.
\end{abstract}

Keywords: Urinary incontinence, Diabetes mellitus, Diabetic neuropathies, Adult, Lower urinary tract symptoms.

\footnotetext{
1 Pontifícia Universidade Católica do Paraná (PUC-PR), Curitiba - PR.

*E-mail: robson.paes@ufpr.br

2 Universidade Federal do Paraná (UFPR), Curitiba - PR.
}

SUBMETIDO EM: 10/2020

ACEITO EM: 11/2020

PUBLICADO EM: 12/2020 


\section{RESUMEN}

Objetivo: Investigar la asociación entre la neuropatía diabética y los síntomas del tracto urinario inferior en personas con diabetes mellitus. Métodos: Revisión integrativa de la literatura, que siguió seis pasos; la búsqueda se realizó en octubre de 2019, en el Portal de la Biblioteca Virtual en Salud y en la Biblioteca Nacional de Medicina, el Sistema de Análisis y Recuperación de Literatura Médica en Línea y las bases de datos Scopus, utilizando los descriptores y operadores booleanos "urinary incontinence" AND "diabetes mellitus" AND "diabetic neuropathies"; Los criterios de inclusión fueron publicaciones de 2009 a 2019 , en portugués, inglés y español. Resultados: De los 52 estudios de la búsqueda, ocho conformaron la muestra final; las mujeres fueron los principales participantes en los estudios; la neuropatía diabética se asoció con síntomas del tracto urinario inferior, con incontinencia urinaria, incontinencia de urgencia, nicturia, vejiga hiperactiva y disfunción sexual femenina; la obesidad, el color blanco, el tiempo y la enfermedad no controlada fueron factores asociados a los síntomas urinarios. Consideraciones finales: La evaluación de la sensibilidad protectora de los pies y la detección temprana de neuropatías ofrece una oportunidad para la prevención de los síntomas del tracto urinario inferior. Las limitaciones de este estudio fueron la heterogeneidad metodológica de los artículos, la estrategia de búsqueda y la falta de datos sobre el género masculino.

Palabras clave: Incontinencia urinaria, Diabetes mellitus, Neuropatías diabéticas, Adulto, Síntomas del sistema urinario inferior.

\section{INTRODUÇÃO}

Diabetes mellitus (DM) é uma doença metabólica crônica, que acomete cerca de 429,9 milhões de pessoas entre 20 e 79 anos em todo o mundo. Caracterizada pela hiperglicemia crônica devido a falha na produção de insulina e/ou aumento da resistência desta nos tecidos. O DM é responsável por altas taxas de mortalidade precoce e complicações, as quais podem ocorrer em nível macrovascular (doença arterial periférica obstrutiva, infarto agudo do miocárdio e acidente vascular cerebral) ou em nível microvascular (neuropatia, retinopatia e nefropatia) (SOCIEDADE BRASILEIRA DE DIABETES, 2019).

A neuropatia diabética (ND) é uma complicação que se constitui por um grupo heterogêneo de manifestações clínicas ou subclínicas, que acometem o sistema nervoso periférico, e que se apresenta de diferentes formas clínicas, mecanismos fisiopatológicos, instalação e evolução. A ND é a complicação microvascular de maior prevalência entre as pessoas com DM; estima-se que a metade da população com a doença possa desenvolver essa disfunção durante a vida (NASCIMENTO OJM, et al., 2016).

Inicialmente a ND causa disfunções motoras seguido de disfunções sensoriais, as quais implicam em síndromes clínicas, a saber: as neuropatias autonômicas e sensoriomotoras (GOLDMAN L e SCHAFER AI, 2018). Na neuropatia sensoriomotora acontece a síndrome neurológica mais comum da doença, a polineuropatia simétrica distal (PSD), que envolve todos os nervos somáticos, principalmente os nervos sensoriais e motores dos pés e das mãos (KAROLI R, et al., 2014). Estas disfunções também podem acontecer em outros órgãos, como na bexiga (LEE WC, et al., 2009).

A disfunção da bexiga diabética (DBD) têm prevalência relatada entre $25 \%$ e $87 \%$ nas pessoas com diabetes mellitus tipo 2 (DM2), apresentando inicialmente hiperatividade detrusora e tardiamente hiposensibilidade e hipoatividade do músculo detrusor (KIRSCHNER-HERMANNS R, et al., 2012).

No início os sintomas de trato urinário inferior (STUI) podem acontecer de forma discreta e sem grandes alterações, fazendo com que as pessoas com DM busquem auxílio profissional quando há agravamento no quadro, como a diminuição da sensibilidade urinária e aumento da capacidade de armazenamento, acarretando em retenção e infecção de trato urinário (ITU) (LEE WC, et al., 2009).

Um estudo de coorte transversal com 102 mulheres com DM2 teve como objetivo investigar a prevalência de DBD e sua relação com outras complicações crônicas da doença, dentre os resultados $36 \%$ apresentavam STUI de moderado a grave, com prevalência de $53 \%$ de bexiga hiperativa, associadas com neuropatia periférica, nefropatia e síndrome metabólica (KAROLI R, et al., 2014). Há uma teoria de que o estresse oxidativo causado pela hiperglicemia pode afetar a musculatura lisa detrusora, e que as alterações macro e microvasculares também são responsáveis por esta complicação (KIRSCHNER-HERMANNS R, et al., 2012). 
O DM é uma doença crônica de alta prevalência na população mundial, de evolução insidiosa e alto risco para múltiplas complicações, sendo necessário pesquisas para identificar os fatores associados a esses agravos. Apesar de conhecidas, as complicações urinárias ainda são pouco investigadas e gerenciadas no sistema de saúde, neste contexto o objetivo desta revisão foi investigar a associação entre a neuropatia diabética e os STUI descritas na literatura nacional e internacional.

\section{MÉTODOS}

Trata-se de uma revisão integrativa da literatura, que seguiu seis etapas. Esse método tem como objetivo analisar os estudos com temas específicos, avaliar criteriosamente as evidências disponíveis e proporcionar a síntese de conhecimento para a incorporação dos resultados na prática clínica (MENDES KDS, et al., 2008).

Na primeira etapa formulou-se a questão de pesquisa baseada no acrônimo das palavras População, Conceito e Contexto (PCC), sendo População: pessoas com DM, Conceito: associação e Comparativo: neuropatia diabética e sintomas de trato urinário inferior, formulando-se a seguinte questão norteadora: existe associação entre neuropatia diabética e os STUI, nas pessoas com DM?

A busca dos estudos ocorreu no mês de outubro de 2019, pelo portal da Biblioteca Virtual de Saúde (BVS) e nas bases de dados da Medical Literature Analysis and Retrieval System Online (Medline), na National Library of Medicine (PubMed) e Scopus utilizando os descritores e operadores booleanos "urinary incontinence" AND "diabetes mellitus" AND "diabetic neuropathies".

Os critérios de inclusão foram estudos originais publicados entre 2009 e 2019, nos idiomas inglês, português e espanhol, que apresentassem de forma quantitativa ou qualitativa a associação entre ND e STUI em adultos.

Os critérios de exclusão foram estudos de revisões, consensos e diretrizes. Na terceira etapa foram removidos os estudos duplicados. Aplicado os filtros e realizado leitura dos títulos e resumos conforme critérios estabelecidos. Selecionado 18 artigos para leitura na integra, dos quais 10 foram excluídos por não abordarem o tema. A amostra final foi composta por oito estudos. Para quarta etapa os autores criaram um roteiro para coleta de dados com os seguintes itens: nome do artigo, autores, ano, país, tipo do estudo, amostragem, objetivos, principais resultados e nível de evidência (OXFORD CENTRE EVIDENCEMEDICINE, 2009).

A sumarização dos resultados, foi realizada na quinta etapa, sendo apresentada de forma descritiva e por meio de quadro. Na sexta etapa foi discutido as evidências encontradas com a síntese do conhecimento para aplicação dos resultados na prática clínica.

\section{RESULTADOS}

$\mathrm{Na}$ busca bibliográfica inicial foram identificados 52 estudos, dos quais oito foram selecionados para a revisão. Todos os artigos escritos por profissionais médicos, sendo sete no idioma inglês e um em espanhol. Utilizado o fluxograma The Preferred Reporting Systematic Reviews and Meta-Analyses of Studies (PRISMA) (GALVÃO TF, et al., 2015) para ilustrar como se procedeu a seleção dos artigos que compuseram a amostra (Figura 1).

Os estudos foram publicados entre 2009 a 2018, sendo três (37,5\%) publicados no ano de 2016. Em relação à metodologia utilizada, dois estudos eram ensaios clínicos randomizados (25\%), dois estudos transversais descritivos (25\%), um prospectivo multicêntrico (12,5\%), um prospectivo de coorte $(12,5 \%)$, um estudo epidemiológico de caso controle (12,5\%) e um transversal prospectivo (12,5\%), com nível de evidência 1B e 2B. O perfil dos estudos, sumarização, apresentação dos resultados e o nível de evidência são apresentadas no Quadro 1.

Os estudos demonstram que os STUI atingem pessoas com DM de ambos os sexos, sendo que cinco $(62,5 \%)$ avaliaram somente mulheres e três $(37,5 \%)$ avaliaram conjuntamente; em relação ao tipo de DM, quatro estudos (50\%) discutiram a questão da IU nas pessoas com DM2, dois (25\%) com DM tipo 1 e 2, dois 
(25\%) não especificaram o subtipo da doença; a idade dos participantes variou entre 19 e 85 anos; o tempo de evolução da DM foi de 5 a 30 anos, sendo a média de 11,8 anos (FAYYAD A, 2009; PHELAN S, et al., 2009; LEE WC e WU HC, 2014; IKEDA M e NOZAWA K, 2015; FURUKAWA S, et al., 2016; JIMÉNESRODRÍGUEZ J, et al., 2016; HOTALING JM, et al., 2016; SAKAKIBARA R, et al., 2018).

Figura 1 - Fluxograma PRISMA para busca, seleção e inclusão dos estudos da revisão integrativa.
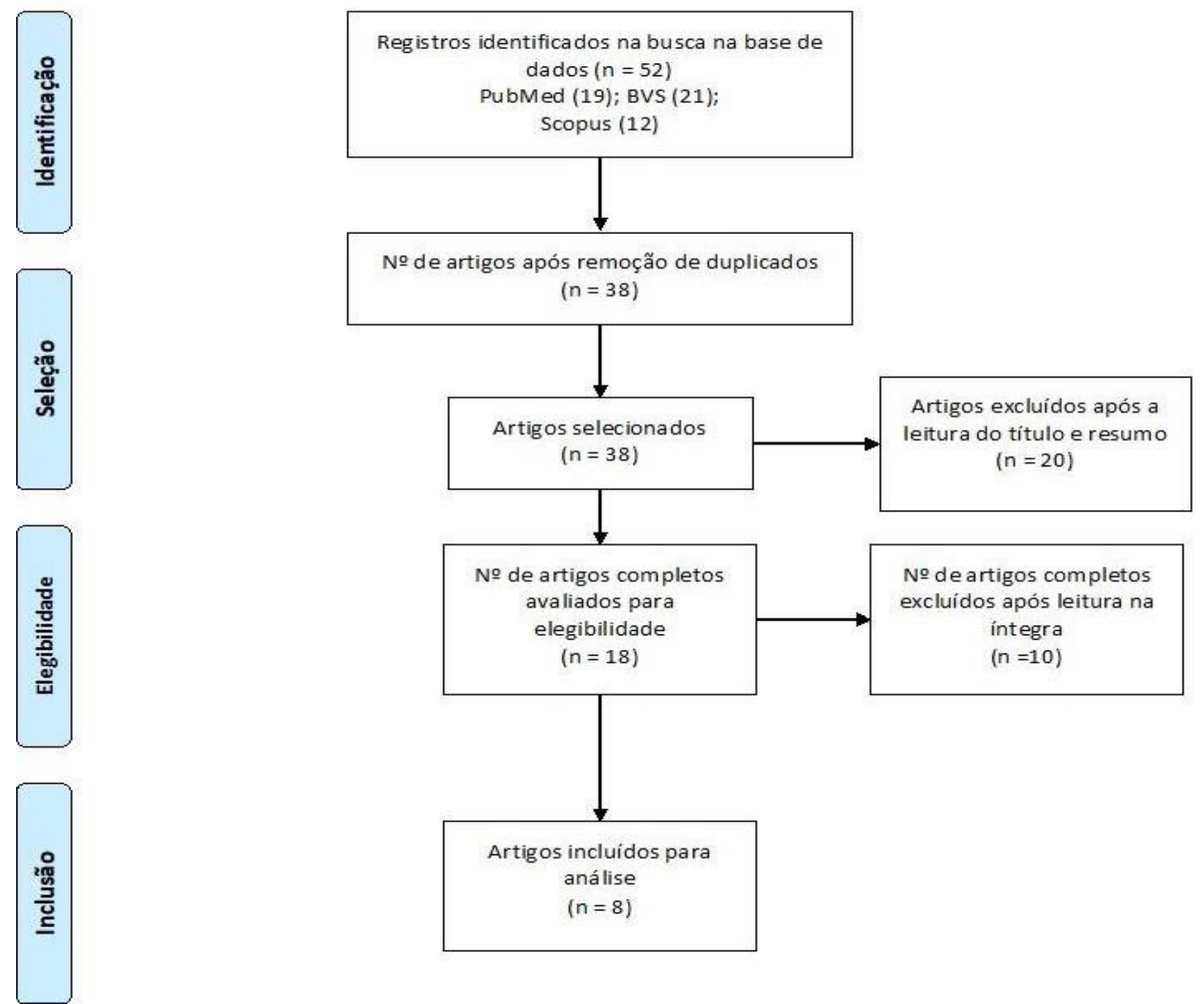

Fonte: Costa EMLM, et al., 2020. Adaptado de Galvão TF, et al., 2015. 
Quadro 1 - Caracterização dos estudos, nível de evidência, sumarização e apresentação dos principais resultados dos estudos selecionados. Curitiba, Paraná, Brasil, 2020

\begin{tabular}{|c|c|c|c|c|c|}
\hline № & Nome do artigo & Autores, ano e país & $\begin{array}{l}\text { Tipo do estudo e } \\
\text { amostragem } \\
\text { Nível de evidência }\end{array}$ & Objetivo & Principais resultados \\
\hline 1 & $\begin{array}{l}\text { Prevalence and risk } \\
\text { factors for bothersome } \\
\text { lower urinary tract } \\
\text { symptoms in women } \\
\text { with diabetes mellitus } \\
\text { from hospital-based } \\
\text { diabetes clinic }\end{array}$ & $\begin{array}{l}\text { FAYYAD A, et al., } 2009 . \\
\text { Reino Unido. }\end{array}$ & $\begin{array}{l}\text { Estudo de Coorte } \\
\text { Prospectivo. } \\
148 \text { participantes. } \\
\text { Nível de evidência 2B }\end{array}$ & $\begin{array}{l}\text { Avaliar fatores de risco } \\
\text { disfunção do trato urinário } \\
\text { inferior (DTUI) e } \\
\text { estabelecer a prevalência } \\
\text { do DTUI em mulheres } \\
\text { com DM. }\end{array}$ & $\begin{array}{l}\text { A DTUI era prevalente nas } \\
\text { mulheres com DM2 e dentre os } \\
\text { sintomas mais citados estavam } \\
\text { urgência, incontinência urinária de } \\
\text { urgência e noctúria. A neuropatia e } \\
\text { hemoglobina glicosilada foram } \\
\text { fatores de risco independentes } \\
\text { para a DTUl }\end{array}$ \\
\hline 2 & $\begin{array}{l}\text { Prevalence and risk } \\
\text { factors for urinary } \\
\text { incontinence in } \\
\text { Overweight and obese } \\
\text { diabetic woman: action } \\
\text { for health in diabetes } \\
\text { (look AHEAD study) }\end{array}$ & $\begin{array}{l}\text { PHELAN S, et al., } 2009 . \\
\text { Estados Unidos da América. }\end{array}$ & $\begin{array}{c}\text { Ensaio clínico randomizado. } \\
2.994 \text { participantes. } \\
\text { Nível de Evidência 1B }\end{array}$ & $\begin{array}{c}\text { Determinar a prevalência } \\
\text { e os fatores de risco para } \\
\text { incontinência urinária (IU) } \\
\text { entre os diferentes } \\
\text { grupos raciais / étnicos } \\
\text { de mulheres com DM2 } \\
\text { com sobrepeso e } \\
\text { obesidade. }\end{array}$ & $\begin{array}{l}\text { A prevalência de IU foi evidenciada } \\
\text { em mulheres obesas e brancas. } \\
\text { Observou-se associação entre a } \\
\text { incontinência urinária de urgência } \\
\text { e neuropatia periférica. }\end{array}$ \\
\hline 3 & $\begin{array}{l}\text { Hyposensitivity of c-fiber } \\
\text { afferents at the distal } \\
\text { extremities as an } \\
\text { indicator of early stages } \\
\text { diabetic bladder } \\
\text { dysfunction in type } 2 \\
\text { diabetic women }\end{array}$ & $\begin{array}{c}\text { LEE WC e WU HC, } 2014 . \\
\text { Taiwan. }\end{array}$ & $\begin{array}{c}\text { Estudo transversal } \\
\text { descritivo. } 137 \text { participantes. } \\
\text { Nível de Evidência } 2 B\end{array}$ & $\begin{array}{l}\text { Avaliar alterações da } \\
\text { função sensorial nas } \\
\text { extremidades distais de } \\
\text { mulheres com DM como } \\
\text { marcador precoce para a } \\
\text { disfunção da bexiga } \\
\text { diabética. }\end{array}$ & $\begin{array}{l}\text { Identificou-se associação entre } \\
\text { hipossensibilidade da função da } \\
\text { fibra } C \text { nas extremidades distais e } \\
\text { disfunção da bexiga diabética em } \\
\text { estágios iniciais nas mulheres com } \\
\text { DM. }\end{array}$ \\
\hline 4 & $\begin{array}{l}\text { Prevalence of } \\
\text { overactive bladder and } \\
\text { its related factors in } \\
\text { Japanese patients with } \\
\text { diabetes mellitus }\end{array}$ & $\begin{array}{l}\text { IKEDA M e NOZAWA K, } 2015 . \\
\text { Japão. }\end{array}$ & $\begin{array}{l}\text { Estudo Prospectivo } \\
\text { transversal. } \\
652 \text { participantes. } \\
\text { Nível de Evidência 1B }\end{array}$ & $\begin{array}{l}\text { Avaliar a prevalência da } \\
\text { bexiga hiperativa e } \\
\text { explorar os fatores } \\
\text { associados com este } \\
\text { evento. }\end{array}$ & $\begin{array}{l}\text { A polineuropatia periférica } \\
\text { apresentou relação positiva com a } \\
\text { bexiga hiperativa. }\end{array}$ \\
\hline
\end{tabular}

REAS/EJCH | Vol.12(12) | e5574 | DOI: https://doi.org/10.25248/reas.e5574.2020 Página $\mathbf{5}$ de 11 


\begin{tabular}{|c|c|c|c|c|c|}
\hline № & Nome do artigo & Autores, ano e país & $\begin{array}{l}\text { Tipo do estudo e } \\
\text { amostragem } \\
\text { Nível de evidência }\end{array}$ & Objetivo & Principais resultados \\
\hline 5 & $\begin{array}{l}\text { Microvascular } \\
\text { complications and } \\
\text { prevalence of urgency } \\
\text { incontinence in } \\
\text { japonesepatiets with } \\
\text { type } 2 \text { diabetes mellitus: } \\
\text { the dogo study }\end{array}$ & $\begin{array}{l}\text { FURUKAWA S, et al., } 2016 . \\
\text { Japão. }\end{array}$ & $\begin{array}{l}\text { Estudo de Coorte } \\
\text { prospectivo multicêntrico. } \\
742 \text { participantes. } \\
\text { Nível de Evidência 1B }\end{array}$ & $\begin{array}{l}\text { Avaliar a associação } \\
\text { entre a neuropatia } \\
\text { diabética e IU. }\end{array}$ & $\begin{array}{l}\text { A neuropatia periférica foi } \\
\text { associada positivamente a IU de } \\
\text { urgência. }\end{array}$ \\
\hline 6 & $\begin{array}{l}\text { Prevalencia de } \\
\text { sintomas urinários em } \\
\text { mujeres com diabetes } \\
\text { mellitus }\end{array}$ & $\begin{array}{l}\text { JIMÉNES-RODRÍGUEZ J, et } \\
\text { al., } 2016 . \\
\text { México }\end{array}$ & $\begin{array}{c}\text { Estudo Transversal } \\
\text { descritivo e observacional. } \\
1169 \text { participantes. } \\
\text { Nível de Evidência 2B }\end{array}$ & $\begin{array}{c}\text { Avaliar e descrever a } \\
\text { prevalência e o impacto } \\
\text { dos sintomas urinários na } \\
\text { qualidade de vida. }\end{array}$ & $\begin{array}{l}\text { Os sintomas urinários estavam } \\
\text { associados com a neuropatia } \\
\text { periférica }(91,1 \%) \text {, principalmente } \\
\text { nas pessoas com mais tempo de } \\
\text { diagnóstico de diabetes. }\end{array}$ \\
\hline 7 & $\begin{array}{l}\text { Cardiovascular } \\
\text { Autonomic Neuropathy, } \\
\text { Sexual Dysfunction, and } \\
\text { Urinary Incontinence in } \\
\text { Women With Type } 1 \\
\text { Diabetes }\end{array}$ & $\begin{array}{l}\text { HOTALING JM, et al., } 2016 . \\
\text { Estado Unidos da América. }\end{array}$ & $\begin{array}{l}\text { Estudo epidemiológico de } \\
\text { caso controle. } 580 \\
\text { participantes. } \\
\text { Nível de Evidência 2B }\end{array}$ & $\begin{array}{c}\text { Avaliar a associação } \\
\text { entre neuropatia } \\
\text { autonômica } \\
\text { cardiovascular e } \\
\text { disfunção sexual feminina } \\
\text { e a IU. }\end{array}$ & $\begin{array}{l}\text { Associações da neuropatia } \\
\text { autonômica cardiovascular (NAC) } \\
\text { com o desenvolvimento de } \\
\text { disfunção sexual feminina (DSF) e } \\
\text { IU. NAC pode ser preditora da } \\
\text { neuropatia autonômica diabética } \\
\text { generalizada. }\end{array}$ \\
\hline 8 & $\begin{array}{l}\text { The Relationship } \\
\text { between Bladder, } \\
\text { Periarterial and Somatic } \\
\text { Neuropathy in Diabetes }\end{array}$ & $\begin{array}{l}\text { SAKAKIBARA R, et al., } 2018 . \\
\text { Japão. }\end{array}$ & $\begin{array}{l}\text { Ensaio clínico randomizado. } \\
110 \text { participantes. } \\
\text { Nível de Evidência 1B }\end{array}$ & $\begin{array}{c}\text { Analisar se a disfunção } \\
\text { da bexiga ocorre sem } \\
\text { neuropatia somática ou } \\
\text { se a disfunção da bexiga } \\
\text { está associada a } \\
\text { hipotensão postural. }\end{array}$ & $\begin{array}{l}\text { A disfunção da bexiga foi } \\
\text { relacionada com neuropatia } \\
\text { somática e periarterial. Preconiza a } \\
\text { avaliação regular do resíduo pós } \\
\text { miccional (RPM). Mostrou também } \\
\text { que a bexiga, as neuropatias } \\
\text { somáticas e periarteriais podem } \\
\text { compartilhar o mesmo processo } \\
\text { patológico, relacionado com as } \\
\text { alterações de hiperglicemia }\end{array}$ \\
\hline
\end{tabular}

Fonte: Costa EMLM, et al., 2020.

REAS/EJCH | Vol.12(12) | e5574 | DOI: https://doi.org/10.25248/reas.e5574.2020 Página 6 de 11 
O estudo 1, investigou 148 mulheres com DM2 sobre problemas pélvicos, DTUI, sintomas vaginais e disfunção intestinal inferior. Dentre os sintomas, $28,8 \%$ das mulheres apresentaram IU de urgência, 38,5\% sintomas de urgência e a $32,4 \%$ noctúria. A neuropatia periférica e a hemoglobina glicada (HbA1c), apareceram como fatores de risco independentes para a disfunção miccional (FAYYAD A, 2009).

No artigo 2, os pesquisadores analisaram as mulheres de diferentes raças (brancas, asiáticas e negras) e etnias (americanas, hispânicas e afro-americanas) com sobrepeso e obesidade. No comparativo as mulheres com índice de massa corporal (IMC) $\geq 35 \mathrm{~kg} / \mathrm{m}^{2}$, apresentaram maiores chances de incontinência urinária de esforço (IUE) do que mulheres não obesas; a frequência de IU semanal foi a mais relatada associada ao DM, sendo maior (32\%) em mulheres brancas não hispânicas, em comparação as afro-americanas (18\%) e asiáticas (12\%).

A IU foi prevalente equiparada a retinopatia, microalbuminúria e neuropatia; outros fatores de risco associados foram a idade avançada, pior estado de saúde, histerectomia prévia, ITU, pós menopausa utilizando terapia hormonal, depressão e tabagismo. A histerectomia prévia estava relacionada a um aumento de 40 a $80 \%$ nas chances de IU (PHELAN S, et al., 2009).

Lee WC e Wu HC (2014) investigaram a relação entre neuropatia periférica simétrica distal e estágios iniciais da disfunção autonômica da bexiga em mulheres com DM2. As participantes com dificuldade de miç̧ão foram submetidas à estudo urodinâmico e teste intravesical do limiar de percepção da corrente neuroseletiva, sendo que $24,8 \%$ apresentaram disfunção urodinâmica da bexiga (DUB) e $19 \%$ a síndrome da bexiga hiperativa; as com hipossensibilidade à fibra $C$ no segundo pododáctilo ou no hálux, também demonstraram maiores taxas de DUB.

Entre as mulheres com DUB positivo, os valores do teste do limiar de percepção da corrente neuroseletiva foram significativamente associados aos valores intravesicais; a hipossensibilidade dos nervos aferentes de fibras $C$ não mielinizados nas extremidades distais é um indicador da DBD em estágios iniciais.

A prevalência de bexiga hiperativa em homens e mulheres com DM, incluindo aqueles com e sem IU, foi investigada no estudo 4, sendo explorada por análise de regressão logística e multivariada que mostrou que a polineuropatia diabética sintomática estava significativamente relacionada a prevalência de bexiga hiperativa (IKEDA M e NOZAWA K, 2015).

Os pesquisadores do estudo 5 , examinaram a associação entre ND, definida por sintomas neuropáticos, ausência do reflexo de Aquiles e/ou percepção anormal de vibração, e incontinência de urgência, sendo diagnosticada nos participantes que apresentaram duas ou mais das características. A IU teve prevalência em $8,6 \%$ dos participantes com associação a ND (FURUKAWA S, et al., 2016).

A prevalência de STUI e o impacto na qualidade de vida (QV) foram avaliados no artigo 6: dentre os STUI a IUE e por urgência foram predominantes, seguidas por sintomas obstrutivos e irritativos. O impacto destes sintomas na QV foi leve à moderado e foram associados com a neuropatia periférica, principalmente nas pessoas com mais tempo de diagnóstico de DM (JIMÉNES-RODRíGUEZ J, et al., 2016).

O estudo 7, avaliou a associação entre NAC, DSF e IU em 580 mulheres com DM1, mostrando associação entre estes por regressão multivariável; houve significância estatística entre as medidas de NAC e complicações do DM, e o subsequente relato de DSF ou UI (HOTALING JM, et al., 2016).

Sakakibara R, et al. (2018) rastrearam a ND em 110 participantes, independentemente de seus sintomas; o tempo médio da doença foi de 14,0 anos e valor médio de $\mathrm{HbA} 1 \mathrm{c}$ de $10,1 \%$. Os resultados apontaram anormalidade da condução das fibras nervosas, volume de diurese pós miccional elevado e hipotensão postural.

O volume anormal de resíduo pós-miccional não estava associado ao valor da HbA1c, mas estava claramente relacionado ao tempo do DM; a disfunção vesical foi correlacionada com neuropatia somática e periarterial; $16 \%$ dos casos de disfunção da bexiga ocorreram em pessoas sem neuropatia somática ou periarterial; sendo sugerido a medição regular do volume residual pós-miccional pois a neuropatia pode ocorrer de forma insidiosa. 


\section{DISCUSSÃO}

A associação da ND com STUI é relevante, mesmo em estudos com diferentes objetivos e abordagens metodológicas, os resultados são muito similares. Diante da análise dos aspectos relevantes da pesquisa, os STUI foram prevalentes nas mulheres com DM, e dentre os sintomas mais citados estavam urgência urinária, incontinência urinária por urgência e a noctúria (FAYYAD A, 2009; PHELAN S, et al., 2009; LEE WC e WU HC, 2014; IKEDA M e NOZAWA K, 2015; FURUKAWA S, et al., 2016; JIMÉNES-RODRÍGUEZ J, et al., 2016; HOTALING JM, et al., 2016; SAKAKIBARA R, et al., 2018).

A neuropatia autonômica resulta em sensação miccional prejudicada, fluxo urinário reduzido e altos volumes pós miccionais. No estudo desenvolvido com 652 participantes de ambos os sexos com DM1 e 2, foi identificado que $43,3 \%$ apresentavam sintomas neuropáticos com retenção de volumes superiores a $50 \mathrm{~mL}$ após a micção, dos quais 11,8\% eram assintomáticos (IKEDA M e NOZAWA K, 2015).

Fayyad A, et al. (2009) concluíram que neuropatia e HbA1c aumentada aparecem como fatores de risco para a disfunção miccional. A detecção e identificação precoce do processo neuropático oferece uma oportunidade para a pessoa com DM controlar os níveis glicêmicos para postergar as complicações da doença (LENHERR SM, et al., 2016).

Estudos eletrofisiológicos têm demonstrado relação direta entre os níveis de $\mathrm{HbA} 1 \mathrm{c}$ e a presença e severidade da ND. Um grande número de mecanismos tem papel no desenvolvimento da ND, como os metabólicos, vasculares, autoimunes, além de deficiências neuro-hormonais e fatores de crescimento. A etiologia das disfunções vesicais associadas ao DM é multifatorial, e pode ser resultado de uma alteração na fisiologia da célula do músculo detrusor, na função do componente neuronal ou urotelial, e processo inflamatório microvascular (OLIVEIRA EG, et al., 2011).

A glicosúria tem efeitos variáveis nos parâmetros urodinâmicos e nos STUI, com associação significativa de ITU e IU, como valores aumentados de HbA1c (JAMES R e HIJAZ A, 2014). O DM de longa data provoca paralisia do músculo detrusor, causando dificuldades na micção ocasionando na ocorrência de cistopatia diabética (HILL SR, et al., 2008). Na população feminina com DM, 50\% apresentam alguma disfunção vesical, resultando nos STUI como urgência urinária, noctúria e IU, causada pela diminuição da contratilidade e aumento do volume residual (KORELO RIG, et al., 2011).

A ITU sintomática e assintomática também é um achado frequente nos estudos realizados nas pessoas com DM de ambos os sexos, pois o descontrole da doença danifica a microvascularização, consequentemente a inervação do esfíncter uretral e o músculo detrusor, causando diminuição da sensibilidade, disfunção e instabilidade da bexiga, retenção e aumento do volume residual de urina, contribuindo para a ocorrência de IU, colonização de bactérias e ITU (BROWN JS, et al., 2006; IZCI Y, et al., 2009; CASARIN N, et al., 2015).

Dados de uma revisão de literatura descrevem que as alterações presentes no músculo e na matriz extracelular uretral estão entre as principais causas de IU de esforço. Os dados sugerem que a IU pode ser a consequência mais comum da hiperglicemia se comparada à outras complicações. Existe também a hipótese de que as ITU recorrentes possam causar o desenvolvimento progressivo da DBD, que então progride com problemas de armazenamento descompensando a função de micção (LIN TL, et al., 2012).

As pessoas com a DM podem apresentar alguma disfunção urinária progressiva de acordo com o estágio da doença. Uma boa triagem inicial destas pessoas é indispensável para detectar e gerenciar os STUI em estágios iniciais (MAJIMA T, et al., 2019). A NB é uma das principais complicações que aparece com o tempo de evolução da doença, sendo caracterizada pela degeneração progressiva dos axônios das fibras nervosas, atreladas as alterações metabólicas, vasculares, autoimunes, deficiências neuro-hormonais e fatores de crescimento (NASCIMENTO OJM, et al., 2016).

A hiperglicemia persistente parece ser o fator causal primário, a qual leva ao acúmulo de produtos da via dos polióis (como sorbitol e frutose) nos nervos, causando lesões por meio de mecanismos ainda desconhecidos. Portanto, a ND pode afetar o sistema nervoso autônomo e o sistema nervoso periférico, desenvolvendo alterações focais ou difusas, proximais ou distais com envolvimento dos neurônios somáticos 
(NASCIMENTO OJM, et al., 2016). As alterações da matriz extracelular causadas pelo DM promovem um impacto sobre a continência urinária sendo julgada como um dos principais fatores, relacionados à incontinência (MAJIMA T, et al., 2019).

A neuropatia autonômica também é umas das condições que levam as alterações de sensação vesical, fluxo urinário reduzido e altos volumes residuais. No estudo desenvolvido com 652 participantes de ambos os sexos com DM1 e 2, os autores identificaram que 43,3\% apresentavam sintomas neuropáticos com retenção de volumes (IKEDA M e NOZAWA K, 2015). A fase inicial da DBD se manifesta como hiperatividade do detrusor, levando a frequência urinária e urgência. Ao longo do tempo, o estresse oxidativo progressivo e a neuropatia levam à descompensação da musculatura do detrusor, levando assim à bexiga hipocontrátil ou atomismo (ZHANG H, et al., 2012).

Lee WC e Wu HC (2014) identificaram a associação entre hipossensibilidade da função da fibra C nas extremidades distais e DBD em estágios iniciais nas mulheres com DM. Sabe-se que estudos eletrofisiológicos permitem identificar a associação entre a hipossenssbilidade da função da fibra $C$ nas extremidades e a DBD em estágios iniciais. Portanto, o comprometimento da fibra C nas extremidades sinalizam a polineuropatia, podendo ser um evento sentinela para a DBD.

A NAC foi umas das complicações resultantes da revisão, ela tem elevada repercussão clínica da doença, e sua prevalência aumenta progressivamente em proporção direta com a idade, a duração da doença e o descontrole controle glicêmico, porém é pouco compreendida e diagnosticada. Ela ocorre quando há uma lesão das fibras autonômicas periféricas (simpático e parassimpático) relacionadas ao sistema cardiovascular, resultando em distúrbios na sua regulação neuro-humoral. Esse tipo de neuropatia pode ser encontrado em aproximadamente $25 \%$ das pessoas com DM1 e 34\% nas com DM2, ressaltando que os sinais e sintomas tardiamente no DM1, e geralmente nesta fase a NAC se encontra em estágios avançados e irreversíveis (ROLIM LCSP, et al., 2008).

A NAC pode predizer a neuropatia autonômica diabética generalizada, sendo um marcador de sensibilidade e indicador clínico para a implementação de estratégias de prevenção e tratamento para esta neuropatia (HOTALING JM, et al., 2016).

O sobrepeso e a obesidade também são fatores que influenciam diretamente nas disfunções vesicais. Um estudo randomizado controlado verificou que as mulheres com IMC $\geq 35 \mathrm{~kg} / \mathrm{m}^{2}$ apresentaram maiores chances de IU de estresse, afirmando que a obesidade é um fator de risco para IU principalmente após a menopausa (PHELAN S, et al., 2009). Em comparação com os outros fatores de risco (idade avançada, raça branca, paridade e número de partos vaginais), a obesidade está intimamente relacionada à perda urinária, além de estar associada a maior morbidade secundária, ao aumento da resistência à insulina, DM, hipertensão e dislipidemias (GUEDES PF, et al., 2017).

As mulheres com obesidade têm maiores chances de desenvolverem IU, especialmente quando relacionada ao esforço (CASTRO LA, et al., 2012). Acredita-se que o excesso de peso aumente a pressão abdominal durante as atividades diárias, o que provoca o aumento da pressão vesical, maior mobilidade da uretra e do colo vesical desenvolvendo a IU (OLIVEIRA E, et al., 2010). Outros fatores como as doenças ginecológicas e a idade avançada podem contribuir para os STUI. As mulheres em idade avançada apresentam maior incidência de déficit funcional, redução da força na musculatura do assoalho pélvico, diminuição das atividades do sistema nervoso e circulatório, redução da elasticidade e contratilidade da bexiga e deficiência esfincteriana (TAMANINI JTM, et al., 2009).

No que se refere à IU no idoso, cabe ressaltar a dificuldade de diagnóstico, tendo em vista que as pessoas leigas e alguns profissionais costumam associar a IU como consequência do processo fisiológico normal do envelhecimento. Neste aspecto é urgente a necessidade de conscientizar as pessoas e os profissionais de que a IU é um problema tratável (TAMANINI JTM, et al., 2009).

A prevalência e os fatores de risco para IU entre mulheres com DM2, obesas, brancas e com neuropatia periférica (PHELAN S, et al., 2009), podem estar associadas as questões etiológicas, multifatoriais e complexas, resultantes da interação de genes, ambiente, estilo de vida e fatores emocionais (ASSOCIAÇÃO BRASILEIRA PARA O ESTUDO DA OBESIDADE E SÍNDROME METABÓLICA, 2016). 
Preconiza-se a avaliação regular do volume RPM nas pessoas com DM, pois a DBD está relacionada com neuropatia somática e periarterial, sendo que estas podem compartilhar o mesmo processo patológico, devido a hiperglicemia (SAKAKIBARA R, et al., 2018). O diagnóstico precoce das alterações urinárias nas mulheres com DM, dão subsídios para o planejamento de cuidados para prevenir e tratar as complicações, melhorando assim a QV destas pessoas (HOTALING JM, et al., 2016).

As limitações deste estudo foram a heterogeneidade metodológica dos artigos e a estratégia de busca. Ressalta-se que há uma lacuna do conhecimento sobre essas complicações do DM na população masculina, assim como a falta de estudos com características epidemiológicas, étnicas e raciais da população brasileira.

\section{CONSIDERAÇÕES FINAIS}

Os artigos analisados apontaram a relação da neuropatia diabética com os sintomas de trato urinário inferior, os quais repercutem negativamente na qualidade de vida. Esses dados alertam os profissionais sobre a importância de avaliar a sensibilidade protetora dos pés, constatar precocemente a neuropatia e investigar os sintomas de trato urinário inferior, pois são frequentemente subestimados; cabe ressaltar a necessidade do incentivo ao paciente para o controle da doença, por meio da adesão as terapias medicamentosas e mudança no estilo de vida, visando reduzir as complicações relacionadas ao diabetes mellitus. Encoraja-se o desenvolvimento de pesquisas nacionais envolvendo esta temática.

\section{REFERÊNCIAS}

1. ASSOCIAÇÃO BRASILEIRA PARA O ESTUDO DA OBESIDADE E DA SÍNDROME METABÓLICA (ABESO) Diretrizes brasileiras de obesidade. 4. ed. São Paulo, 2016.

2. BROWN, JS, et al. Prevalence and risk factors for urinary incontinence in women with type 2 diabetes and impaired fasting glucose: findings from the National Health and Nutrition Examination Survey (NHANES) 2001-2002. Diabetes care, 2006; 29(6): 1307-1312.

3. CASARIN N, et al. O diabete mellitus nas disfunções pélvicas femininas. Fisioterapia Brasil, 2015; 16(1): 61-67.

4. CASTRO LA, et al. Efeitos da cirurgia bariátrica na função do assoalho pélvico. Arquivos brasileiros de cirurgia digestiva, 2012; 25(4): 263-268.

5. FAYYAD A, et al. Prevalence and risk factors for bothersome lower urinary tract symptoms in women with diabetes mellitus from hospital-based diabetes clinic. International Urologynecology Journal, 2009: 20(11): 1339-1344.

6. FURUKAWA S, et al. Microvascular complications and prevalence of urgency incontinence in Japanese patients with type 2 diabetes mellitus: The dogo study. Neurourol Urodyn, 2016: 35(8): 1024-1027.

7. GALVÃO TF, et al. Principais itens para relatar Revisões sistemáticas e Meta-analises: A recomendação PRISMA. Epidemiologia e Serviços de Saúde, 2015; 24: 335-342.

8. GOLDMAN L, SCHAFER Al. Goldman-Cecil Medicina: Adaptado à realidade brasileira. Elsevier Brasil, 2018.

9. GUEDES PF, et al. Sobrepeso e obesidade em mulheres com incontinência urinária e a repercussão na qualidade de vida. Disciplinarum Scientia: Saúde, 2017; 18(3): 539-550.

10. HILL SR, et al. Diabetes mellitus and demale lower urinary tract symptoms: a review. Neurourol Urodyn, 2008; 27(5): 362-367.

11. HOTALING JM, et al. Cardiovascular autonomic neuropathy, sexual dysfunction, and urinary incontinence in women with type 1 diabetes. Diabetes Care, 2016; 39(9): 1587-1593.

12. IKEDA M, NOZAWA K. Prevalence of overactive bladder and its related factors in Japanese patients with diabetes mellitus. Endocr J, 2015; 62(9): 847-854.

13. $\mathrm{IZCl} \mathrm{Y}$, et al. The association between diabetes mellitus and urinary incontinence in adult women. International Urogynecology Journal, 2009; 20(8): 947-952.

14. JAMES R, HIJAZ A. Lower urinary tract symptoms in women with diabetes mellitus: a current review. Curr Urol Rep, 2014; 15(10): 440-447.

15. JIMÉNEZ-RODRÍGUEZ J, et al. Prevalence of urinary tract symptoms in women with diabetes mellitus. Revista Médica del Instituto Mexicano del Seguro Social, 2016; 54(1): 70-74.

16. KAROLI R, et al. A study of bladder dysfunction in women with type 2 diabetes mellitus. Indian Journal of Endocrinology and Metabolism, 2014; 8(4): 552-557.

17. KIRSCHNER-HERMANNS R, et al. Does diabetes mellitus-induced bladder remodeling affect lower urinary tract function? ICI-RS 2011. Neurourol Urodyn, 2012; 31(3): 359-364.

18. KORELO RIG, et al. Influência do fortalecimento abdominal na função perineal, associado ou não à orientação de contração do assoalho pélvico, em nulíparas. Fisioterapia em Movimento, 2011; 24(1): 75-85.

19. LEE WC, WU HC. Hyposensitivity of C-fiber afferents at the distal extremities as an indicator of early stages diabetic bladder dysfunction in type 2 diabetic women. PLoS one, 2014; 9(1): e86463.

20. LEE WC, et al. Investigation of orodynamic characteristics and bladder sensory function in the early stages of diabetic bladder dysfunction in women with type 2 diabetes. The Journal of Urology, 2009; 181(1): 198-203. 
21. LENHERR SM, et al. Glycaemic control and risk of incident urinary incontinence in womem with type 1 diabetes: results from the diabetes control and complications trial and epidemiology of diabetes interventions and complications (DCCT/EDIC) study. Diabet Med, 2016; 33(11): 1528-1535.

22. LIN TL, et al. Aging and recurrent urinary tract infections are associated with bladder dysfunction in type 2 diabetes. Taiwanese Journal of Obstetrics and Gynecology, 2012; 51(3): 381-386.

23. MAJIMA T, et al. Urodynamic analysis of the impact of diabetes mellitus on bladder function. International Journal of Urology, 2019; 26(6): 618-622.

24. MENDES KDS, et al. Revisão integrativa: método de pesquisa para a incorporação de evidências na saúde e na enfermagem. Texto Contexto Enferm, 2008; 17(4): 758-764.

25. NASCIMENTO OJM, et al. Neuropatia Diabética. Rev Dor, 2016; 17: 46-51.

26. OLIVEIRA E, et al. Influência de índice de massa corporal na incontinência urinária feminina. Revista Brasileira de Ginecologia e Obstetrícia, 2010; 32(9): 454-458.

27. OLIVEIRA EG, et al. Diabete melito como fator associado às disfunções do trato urinário inferior de mulheres atendidas em serviço de referência. Revista Brasileira de Ginecologia e Obstetrícia, 2011; 33(12): 414-420.

28. OXFORD CENTRE FOR EVIDENCE-BASED MEDICINE, $2009 . \quad$ Disponível em: https://www.cebm.ox.ac.uk/resources/levels-of-evidence/oxford-centre-for-evidence-based-medicine-levels-ofevidence-march-2009. Acesso em: 04 out. 2020.

29. PHELAN S, et al. Prevalence and risk factors for urinary incontinence in overweight and obese diabetic women action for health in diabetes (look AHEAD) study. Diabetes Care, 2009; 32(8): 1391-1397.

30. ROLIM LCSP, et al. Neuropatia autonômica cardiovascular diabética: fatores de risco, impacto clínico e diagnóstico precoce. Arq. Bras. Cardiol, 2008; 90(4): e24-e32.

31. SAKAKIBARA R, et al. The relationship between bladder, periarterial and somatic neuropathy in diabetes. Internal Medicine, 2018; 57(15): 9749-9752.

32. SOCIEDADE BRASILEIRA DE DIABETES. Diretrizes da Sociedade Brasileira de diabetes: 2019-2020. São Paulo, 2019.

33. TAMANINI JTN, et al. Análise da prevalência e fatores associados à incontinência urinária entre idosos do munícipio de São Paulo, Brasil: estudo SABE (Saúde, Bem-estar e Envelhecimento). Cad. Saúde Pública, 2009; 25(8): 17561762.

34. ZHANG $\mathrm{H}$, et al. Adipose tissue-derived stem cells ameliorate diabetic bladder dysfunction in a type II diabetic rat model. Stem cells and development, 2012; 21(9): 1391-1400. 